\title{
Direct view of a dark and distant world
}

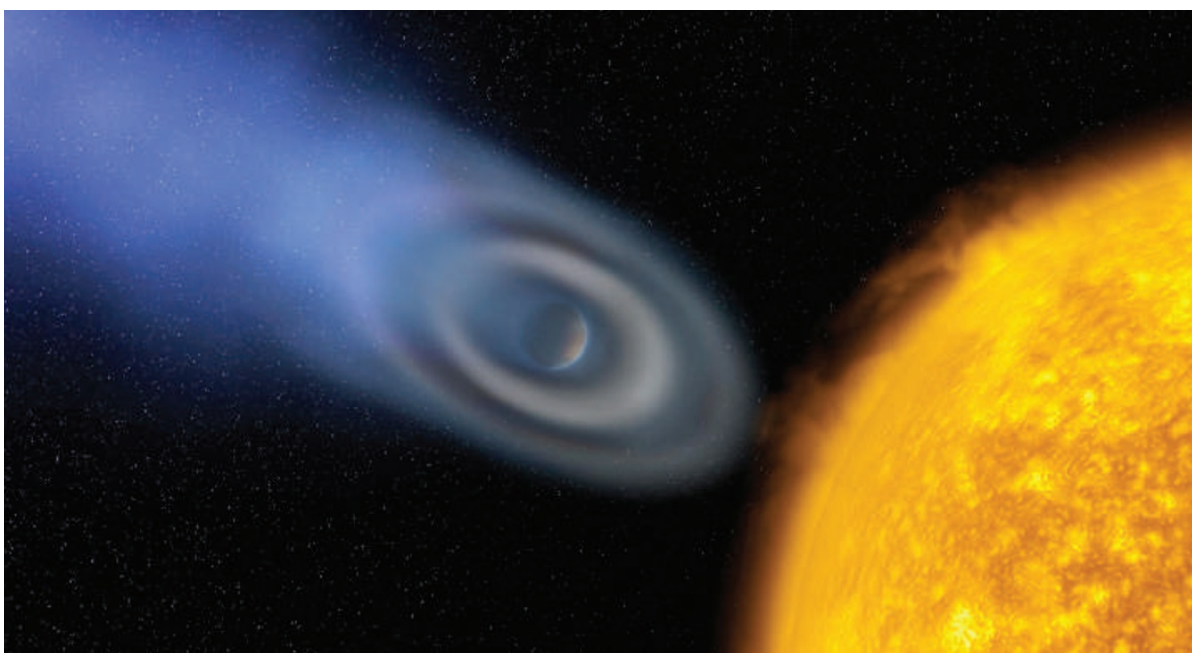

The contents of exoplanet HD 209458b's atmosphere remain controversial.

Three groups of astronomers have made the first detailed measurements of radiation emitted by two exoplanets. Although sketchy, the results hint that the worlds are cloaked in black silicate clouds that don't let light in or out.

After a bitter race, Jeremy Richardson at NASA's Goddard Space Flight Center in Greenbelt, Maryland, and his colleagues are the first to publish their results (see 'Crossing the line'). They used the Spitzer Space Telescope to analyse the atmosphere of a hot Jupiter-like planet called HD 209458b, 150 light years from Earth, and announce their results in this week's Nature (see page 892 ).

Previous studies used the Hubble Space Telescope to look for wavelengths of starlight that are absorbed when HD $209458 \mathrm{~b}$ passes in front of its parent star, and have deduced the presence of sodium, as well as possibly carbon and oxygen, in its atmosphere (D. Charbonneau et al. Astrophys. J. 568, 377-384; 2002; A. VidalMadjar et al. Astrophys J. 604, L69-L72; 2004).

But Richardson's group used the Spitzer Space Telescope to detect the planet's atmosphere directly, by comparing the infrared radiation from the star and planet together, with that emitted when the planet was behind the star.

Molecules absorb and emit infrared radiation at specific wavelengths when they vibrate, and the researchers had expected the planet's infrared spectrum to show up water and carbon dioxide, as well as carbon-containing molecules such as methane. But Richardson says that the spectrum was flat, showing nothing apart from a peak he attributes to silicates, and possibly a molecule containing carbon-carbon bonds such as those seen in benzene.
The absence of water was particularly surprising because gas giants such as HD 209458b are predicted to produce large quantities of water. "Theorists will tell you that water has to be there," Richardson says. He thinks that the silicates might provide an explanation. Previous observations of the planet have suggested the presence of high clouds, so it's possible that the silicate clouds are blocking the water, and other chemicals, from Spitzer's gaze.

Carl Grillmair at the California Institute of Technology in Pasadena and his colleagues used Spitzer to measure the atmospheric spectrum of a similar planet 60 light years from Earth, called HD 189733b. They weren't far behind Richardson in their analysis, and their full results will soon be unveiled in the Astrophysical Journal.

Grillmair's group also got a flat spectrum, with no water, methane or carbon dioxide. The team didn't see silicates either, but co-author David Charbonneau from the Harvard-Smithsonian Center for Astrophysics in Cambridge, Massachusetts, says that Richardson's result offers a tantalizing clue to what might be going on. "We find it hard to believe that there isn't water because it is so easy to make," he says.

Mark Swain at the Jet Propulsion Laboratory, Pasadena, California, offers a third perspective. He started working on Richardson's data once they were made public a year after they were collected, and has submitted his results to the Astrophysical Journal. For now he is keeping the details to himself, but he says that he didn't see any water or silicates and isn't convinced by Richardson's idea of silicate clouds. "That's not a conclusion I would sign up to," he says.

All three groups have applied to make followup observations with Spitzer. Theorist Alan Boss of the Carnegie Institute in Washington DC says that it's not surprising that the researchers didn't see what they expected first time round. In exoplanet research, with so few data to go on, theory must follow observations as they are made, he points out. "It would have been a miracle if theorists had been able to predict what the spectrum of these two hot Jupiters would be like." Katharine Sanderson

\section{Crossing the line}

The debate over the presence of silicates isn't the only controversy the work has triggered. Astronomers are also discussing the race that led up to this week's publication on HD 209458b.

Jeremy Richardson and his colleagues from NASA's Goddard Space Flight Center in Greenbelt, Maryland, used the Spitzer telescope to make measurements of the planet in July 2005. By Spitzer rules, they had sole use of the data for 12 months, at which point the data were made publicly available.

Mark Swain from the Jet Propulsion Laboratory in
Pasadena, California, then began his own analysis of the data. But even though he started a year behind, he was quicker, and Richardson says that he had to rush to publish first. "It was certainly a time to redouble my efforts," he says. Richardson isn't happy about the attempt to scoop him, especially for something as high profile as being first to measure an exoplanet's atmosphere. If you simply want to contribute to a fastmoving community, then using someone else's data makes sense, he says. But he warns that it might not be the right approach if the goal is to establish working collaborations with members of the scientific community.

Swain says that he is surprised that Richardson's analysis took so long. "I expected they would write the paper before me," he says.

The race has raised a few eyebrows, but most astronomers seem to agree the most important thing is to get the results out fast. "It isn't bad form," says Geoffrey Marcy, from the University of California, Berkeley, who discovered 121 of the 209 exoplanets found so far. "Spitzer is expensive and we want the best results." K.S. 